\title{
LYMAN EDGES IN QUASAR SPECTRA
}

\author{
A.L. Kinney, R.R.J. Antonucci, and H.C. Ford
}

Space Telescope Science Institute, Baltimore, MD

\begin{abstract}
This is a study of the Lyman edge region in the spectra of eleven high redshift quasars. We present large aperture, low resolution data designed to detect broadened Lyman edge absorption predicted by thermal models of the Big Blue Bump continuum component. We also present high resolution data on the edge regions and the Lyman alpha emission line for nine of the objects. We show some partial absorption edges and discuss whether or not they can be interpreted as support for the accretion disk model.
\end{abstract}

\section{DISCUSSION}

There are two reasons to study the Lyman edge region in quasar spectra. The behavior in that region should be a powerful diagnostic of the emission mechanism, and it should reveal the presence of absorbing material such as Broad Emission Line clouds in the line of sight.

The Big Blue Bump component extends from around 1 micron past $1000 \AA$, and in some cases apparently all the way to the soft $X$-ray region. Energetically it is very important and possibly dominant. The leading explanation, based on continuum spectral fits, is thermal radiation from a geometrically thin, optically thick accretion disk (Malkan 1983). However, standard disk models predict enormous Lyman absorption edges at the systemic redshift, which are apparently not seen. Lowering the surface gravity weakens the predicted absorption (Sun and Malkan, 1988). However, as the radiation pressure limit is approached the disk becomes thick, so the model must be modified.

It may be possible to reduce the predicted edges by invoking a more isothermal atmosphere, either by distributed deposition of accretion energy or X-ray heating from above. The second possibility changes the model rather profoundly, in that the energy no longer derives directly from accretion. Furthermore, such external heating could also drive the edge into emission. In ANY optically thick thermal model some finite absorption or emission edge must occur. Qualitatively, this applies to thick disks and any other configuration as well. For $\approx 30,000 \mathrm{~K}$ gas the opacity changes so enormously at $912 \AA$ that SOME feature should be produced. The "Lyman Edge Test" is arguably the most fundamental, interpretable discriminent between thermal and nonthermal models.

This is a study of the Lyman edge region in the spectra of eleven high redshift quasars. We present large aperture, low resolution data designed to detect broadened Lyman edge absorption predicted by thermal models of the Big Blue Bump continuum component. We also present high resolution data on the edge regions and the Lyman alpha emission line for nine of the objects.

Between three and five objects have broadened, partial edges as expected for thermal models, (S5 0014+813, and 1159+123, Figures 1 and 2, and also 0302-003, S4 0636+680 and 0642+449) but there are several reasons we are not confident of this interpretation. All three definite cases have redshifts relative to the systemic velocities, a possible but unlikely occurance for thin accretion disks. An explanation which is fundamental to the emission mechanism implies that the edges should be fairly universal, especially because our data can show even highly broadened edges. We have some objects with no edges near systemic. Furthermore, Kinney et al.(1988, and in prep) find NO partial 
systemic edges in the spectra of $22 z<0.8$ quasars with reasonably good IUE data.

In one case we can identify narrow Lyman and metal absorption lines with the edge absorption, ruling out an accretion disk, where absorption lines would be broadened beyond recognition, but in other cases the situation is unclear. None of the spectra recover below the partial absorption edges, suggesting partial covering by opaque broad emission line clouds as the cause, but the wavelength interval observable below the edge is generally small. The best way to test whether the partial edges are due to accretion disks appears to be checking for associated narrow absorption lines.

Our spectra show five complete intervening Lyman Limit System edges, and they generally appear to be quite broad. The broadening can perhaps be explained by overlapping high-n absorption lines if the systems are clustered over a range of a few hundred kilometers

1. Kinney, A.L., Bohlin, R.C., Neill, J.D., Blades, J.C., and York, D.G., 1988, Proceedings, of the 10th Year IUE Conference, (Greenbelt, Maryland, April 1988), in press.

2. Malkan, M.A. 1983 Ap. J., bf 268, 582.

3. Sun, W.-H. and Malkan, M.A. 1988, Proceedings, of the 10th Year IUE Conference, (Greenbelt, Maryland, April 1988), in press.

Figures 1 - 2. a) Large aperture, low resolution spectrum. The systemic edge position for the adopted redshift is marked. b) Small aperture, high resolution spectrum of the systemic Ly edge region, with the systemic edge position indicated. c) Small aperture, high resolution spectrum of the Ly alpha region, with the adopted Ly alpha peak marked.
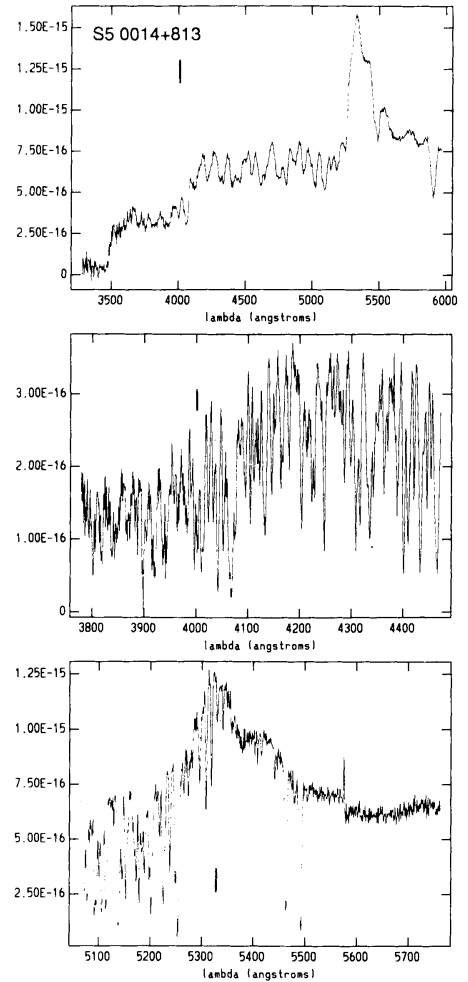
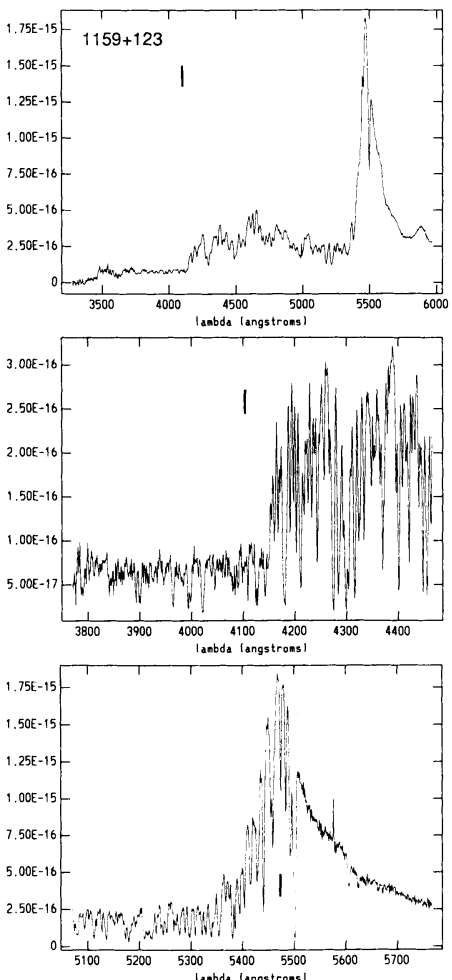